\title{
Morphological Changes in the Ligamentum Flavum in Degenerative Lumbar Canal Stenosis: A Prospective, Comparative Study
}

\author{
Devanand Hulmani ${ }^{1}$, Bhavuk Garg ${ }^{1}$, Nishank Mehta ${ }^{1}$, \\ Asit Ranjan Mridha ${ }^{2}$, Tapas Chandra $\mathrm{Nag}^{3}, \mathrm{Kamran}$ Farooque ${ }^{4}$ \\ ${ }^{1}$ Department of Orthopaedics, All India Institute of Medical Sciences, New Delhi, India \\ ${ }^{2}$ Department of Pathology, All India Institute of Medical Sciences, New Delhi, India \\ ${ }^{3}$ Department of Anatomy, All India Institute of Medical Sciences, New Delhi, India \\ ${ }^{4}$ Department of Orthopaedics, Jai Prakash Narayan Apex Trauma Centre, New Delhi, India
}

Study Design: Prospective, comparative.

Purpose: To compare the histopathological and electron microscopic changes in the ligamentum flavum (LF) between degenerative lumbar canal stenosis (LCS) and lumbar disk herniation (LDH).

Overview of Literature: The LF has been implicated as a key structure in the pathogenesis of LCS. With aging, the LF undergoes morphological changes-a decrease in the elastic component and an increase in the collagen component, in addition to other focal changes. By comparing the histopathological and electron microscopic picture of the LF in elderly patients with LCS with that in young patients with LDH, the role of this ligament in the pathogenesis of LCS may be clarified.

Methods: Forty patients were prospectively recruited and divided into two groups: group 1 included 20 patients with degenerative LCS aged $>55$ years and group 2 included patients with LDH aged $<35$ years. The ligament flava were collected during the patients' surgery. The features noted on histopathological examination included the fibrosis score, the loss of elastic fibers, calcification, chondroid metaplasia, mucinous degeneration, vascularization, long septa, clefts, granulation tissue, and ganglion-like cysts. The features noted on electron microscopic examination included the elastic fiber thickness, the quality of elastic fibers, the elastic:collagen ratio, calcification, melanin fibers, remnants of necrotic cells, and electron-dense material in the LF. All parameters were compared between group 1 and group 2 .

Results: On histopathological examination, the two groups exhibited significant differences regarding three parameters: chondroid metaplasia, long septa, and ganglion-like cysts. On electron microscopy examination, significant differences were observed between the two groups regarding two parameters: the quality of elastic fibers and the elastic:collagen ratio.

Conclusions: Characteristic morphological changes may be noted on histopathological and electron microscopic examination that mark the degenerative changes in the LF that contribute to the occurrence and pathogenesis of degenerative LCS.

Keywords: Degenerative intervertebral disks; Electron microscopy; Histopathology; Ligamentum flavum; Lumbar region

Received Feb 2, 2020; Revised Feb 25, 2020; Accepted Mar 1, 2020

Corresponding author: Bhavuk Garg

Department of Orthopaedics, All India Institute of Medical Sciences, New Delhi, India

Tel: +91-9868397116, Fax: +91-1126594322,E-mail: drbhavukgarg@gmail.com 


\section{Introduction}

Lumbar canal stenosis (LCS) is a common condition in the elderly and is the most common indication for spinal surgery in patients above 65 years of age [1]. While the exact prevalence of LCS is unknown, a study performed in Japan found that its incidence increases with advancing age, with a $10.3 \%-11.2 \%$ incidence in the $70-79$ years of age group compared with a $1.7 \%-2.2 \%$ incidence in the 40-49 years of age group [2].

In 1913, Elsberg [3] first reported hypertrophy of the ligamentum flavum (LF) as a cause of low-back pain or sciatica. Since then, the LF has been implicated as a key structure in the pathogenesis of LCS [4-6]. The normal LF is a well-defined elastic structure that is composed of roughly $80 \%$ elastic fibers and $20 \%$ collagen fibers [7]. It shows uniform morphological characteristics throughout its length, with the exception of close proximity to the bone attachment, where the ligamentous tissue contains elastic fibrocartilaginous tissue-an abundant collagenous component with scattering of chondrocyte-like cells and a few thin elastic fibers. The remainder of the ligament is an almost pure elastic tissue consisting of large elastic fibers, thin bundles of collagen fibers, and fibrocyte-like cells [8]. With aging, the LF undergoes certain pathophysiological changes-a decrease in the elastic component and an increase in the collagen component. In addition, other focal lesions, such as chondroid metaplasia, areas of fibrosis, intra-ligamentous cysts, and hematoma, have also been reported [5,9-11].

Considering the importance of LF in the pathogenesis of LCS, the literature on its histological and microscopic changes during degenerative LCS is scarce. More knowledge of this aspect will provide a greater insight into the exact role of the LF in the pathogenesis of LCS and may help set up a foundation for novel therapies that target the molecular pathways that lead to these pathological changes. Most of the studies available have been carried out over two decades back, thus predating the development of higher-resolution modalities and newer histological and electron microscopic techniques. The purpose of the present study was to evaluate the advanced histopathological and electron microscopic changes in the LF in patients with degenerative LCS compared with young patients with disk herniation. Here, we attempted to answer the following questions. (1) What characteristic histopathological changes are unique to the LF in degenerative LCS com- pared with a non-degenerative pathology, such as lumbar disk herniation (LDH)? (2) What characteristic electron microscopic changes in the LF are unique to degenerative LCS compared with a non-degenerative pathology, such as $\mathrm{LDH}$ ?

\section{Materials and Methods}

The present study was a comparative, observational cohort study of 40 patients who were prospectively recruited and divided into two groups: group 1 included 20 patients with degenerative LCS aged $>55$ years and group 2 included 20 patients with LDH aged $<35$ years. Informed consent was obtained from all study participants and prior approval for the study was obtained from the Institutional Ethics Committee of All India Institute of Medical Sciences, New Delhi (Ref no., IECPG-681/19.01.2017). Patients with infective, malignant, metabolic, or inflammatory disorders of the spine or acute traumatic spine conditions were excluded from the study.

All cases were operated by one of the two orthopedic spine surgeons who are co-authors of the present study (B.G. and K.F.). Patients underwent either only decompression or decompression with instrumented fusion, as deemed appropriate by the operating team based on the patient's symptomatology and imaging findings. The ligamentum flava were collected during the surgery. While every effort was made to remove the LF en-bloc, in most cases, the LF was removed piecemeal. For histopathological examination, the sample was transported in $10 \%$ neutral buffered formalin, whereas for electron microscopic examination, it was sent in $2.5 \%$ glutaraldehyde and $2 \%$ paraformaldehyde in $0.1 \mathrm{M}$ sodium phosphate buffer $(\mathrm{pH}$ 7.3).

For histopathological evaluation, the samples were fixed in $10 \%$ neutral buffered formalin for 48 hours and embedded into a paraffin block. Sections of approximately $5 \mu \mathrm{m}$ were cut, deparaffinized, and stained using routine hematoxylin and eosin staining. Additional histochemical stains used for the evaluation of morphological alteration included: (1) Masson's trichrome staining for evaluating the degree of fibrosis, (2) Verhoeff-Van Gieson elastic staining for elastic fibers, (3) von Kossa and Alizarin red staining for calcification, and (4) Alcian blue-periodic acid-Schiff staining for chondroid metaplasia and mucinous degeneration.

For transmission electron microscopy, the samples 
were cut into pieces of $1-2 \mathrm{~mm}$ and fixed in $2.5 \%$ glutaraldehyde and $2 \%$ paraformaldehyde in $0.1 \mathrm{M}$ sodium phosphate buffer ( $\mathrm{pH} 7.3$ ) for 12 hours at $40^{\circ} \mathrm{C}$. After washing in buffer, the samples were post-fixed in $1 \%$ osmium tetroxide $\left(\mathrm{OsO}_{4}\right)$ for 1 hour at $40^{\circ} \mathrm{C}$. The samples were dehydrated in an ascending grade of acetone and infiltrated and embedded in araldite CY 212 (TAAB, Aldermaston, UK). Thick sections $(1 \mu \mathrm{m})$ were cut on an ultramicrotome, mounted onto glass slides, stained with aqueous toluidine blue, and observed under a light microscope for gross observation of the area and quality of the tissue fixation. For electron microscopic examination, thin sections of gray-silver color interference (70-80 $\mathrm{nm}$ ) were cut and mounted onto 300 mesh-copper grids. Sections were stained with alcoholic uranyl acetate and alkaline lead citrate, washed gently with distilled water, and observed under a Morgagni 268D transmission electron microscope (Fei Co., Eindhoven, The Netherlands) at an operating voltage of $80 \mathrm{kV}$. Images were digitally acquired using a CCD camera (Mega View III, Fei Co.) attached to the microscope.

Several randomly chosen slides were studied as part of the histopathological examination, and the following parameters were noted on the best representative slide: (1) Fibrosis score: Fibrosis was evaluated semi-quantitatively with respect to the entire area of the tissue examined and graded with reference to its severity (range, $0-4$ ). Grade 0 indicates normal tissue showing no fibrotic regions; grade 1 , fibrosis in $0 \%-25 \%$ of the entire area; grade 2 , fibrosis in $25 \%-50 \%$ of the entire area; grade 3 , fibrosis in $50 \%-$ $75 \%$ of the entire area; and grade 4 , fibrosis in $>75 \%$ of the entire area of the LF examined. (2) Percentage of loss of elastic fibers: The loss of elastic fibers was also evaluated semi-quantitatively with respect to the entire area of the tissue and measured in terms of percentage. (3) Percentage of calcification: Calcification was also assessed semiquantitatively with respect to the entire area of the tissue and measured in terms of percentage. (4) Areas of chondroid metaplasia (presence or absence) were evaluated by Alcian blue staining.

Several randomly chosen fields were studied as part of the electron microscopic evaluation, and the following parameters were evaluated on the best representative field: (1) thickness of the elastic fibers; (2) elastic:collagen ratio (the images acquired during the electron microscopic examination using a CCD camera attached to the microscope were analyzed using the ImageJ software (National
Institutes of Health, Bethesda, MD, USA) for the calculation of the thickness of the elastic fibers and the area of the elastic and collagen fibers. The ratio of elastic fibers to collagen fibers was calculated); (3) quality of the elastic fibers (in terms of their thinning, fragmentation, or degeneration); and (4) calcification (evaluated for its presence or absence).

All parameters were compared between group 1 and group 2. The distribution of the data for two parameters, i.e., the percentage of loss of elastic fibers (a histological parameter) and the elastic:collagen ratio (an electron microscopy parameter), was not normal; hence, the MannWhitney test was used to analyze these parameters. For all other parameters, the chi-square test was used. Significance was set at $p<0.05$.

\section{Results}

A total of 40 patients were recruited into the study and were divided into two study groups. Group 1 consisted of patients with degenerative LCS aged $>55$ years and group 2 consisted of patients with LDH aged $<35$ years. The mean age of the patients with degenerative LCS was $63.50 \pm 6.90$ years (range, $56-83$ years) and that of the LDH

Table 1. Comparison of baseline characteristics between the two study groups

\begin{tabular}{|c|c|c|}
\hline Characteristic & $\begin{array}{c}\text { Degenerative } \\
\text { lumbar canal } \\
\text { stenosis (group 1) }\end{array}$ & $\begin{array}{l}\text { Disc } \\
\text { herniation } \\
\text { (group 2) }\end{array}$ \\
\hline Age (yr) & $63.50 \pm 6.902$ & $26.85 \pm 5.122$ \\
\hline Female:male & $11: 9$ & 10:10 \\
\hline Body mass index $\left(\mathrm{kg} / \mathrm{m}^{2}\right)$ & $25.360 \pm 2.14$ & $23.40 \pm 2.4705$ \\
\hline \multicolumn{3}{|l|}{ Level of involvement } \\
\hline L3-L4 & 1 & 0 \\
\hline L4-L5 & 4 & 9 \\
\hline L5-S1 & 1 & 8 \\
\hline L4-L5, L5-S1 & 7 & 2 \\
\hline L3-L4, L4-L5 & 4 & 0 \\
\hline L3-L4, L4-L5, L5-S1 & 2 & 1 \\
\hline L2-L3, L3-L4, L4-L5 & 1 & 0 \\
\hline \multicolumn{3}{|l|}{ Surgical procedure } \\
\hline Decompression only & 1 & 17 \\
\hline $\begin{array}{l}\text { Decompression+transforaminal } \\
\text { lumbar interbody fusion }\end{array}$ & 19 & 3 \\
\hline
\end{tabular}

Values are presented as mean \pm standard deviation or number. None of the above parameters were statistically significant. 

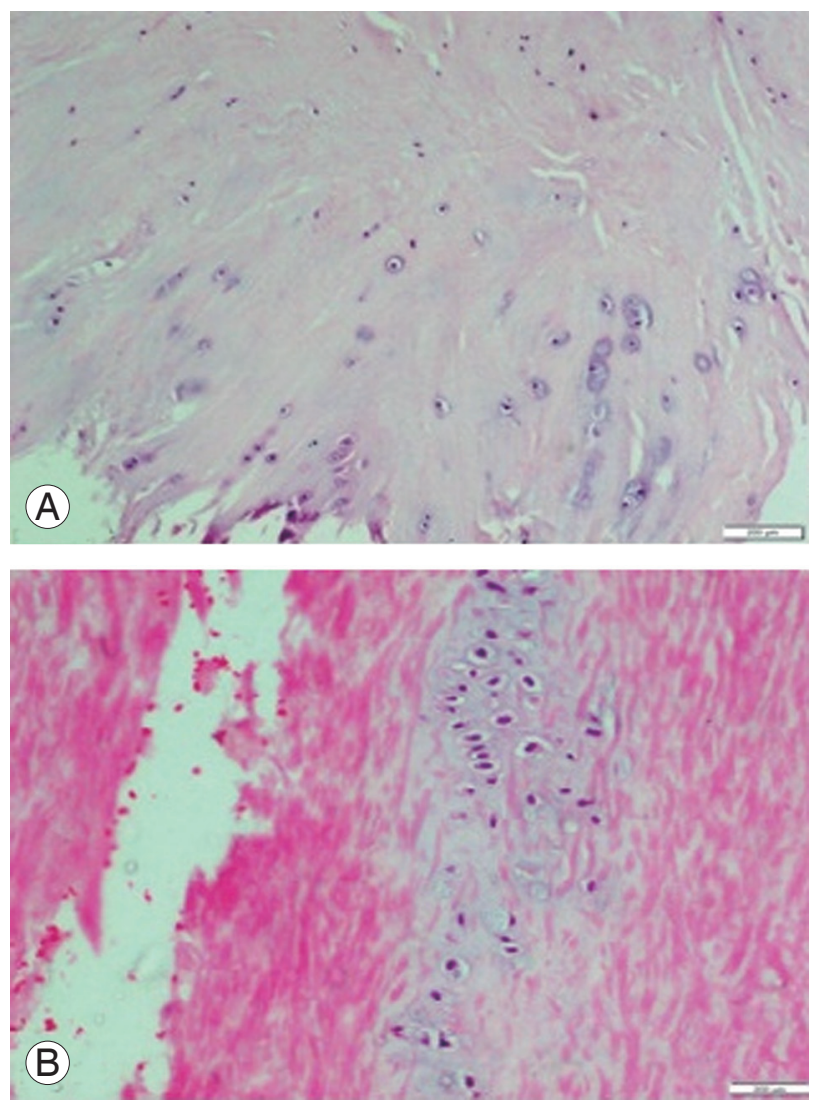

Fig. 1. (A) HE stained section of ligamentum flavum in patient with degenerative lumbar canal stenosis showing areas of chondroid metaplasia at bony attachment. Chondrocytes arranged in pairs or in chains or clusters (H\&E, $\times 100)$. (B) HE stained section of ligamentum flavum of a patient with disc herniation showing areas of chondroid metaplasia in the central region $(H \& E, \times 200)$. $H E$, hematoxylin and eosin.

group was $26.85 \pm 5.12$ years (range, $18-34$ years). Table 1 lists the relevant demographic details of the two groups.

Various histopathological parameters, such as fibrosis, percentage of loss of elastic fibers, percentage of calcification, presence of chondroid metaplasia, mucinous degeneration, vascularization, long septa, clefts, granulation tissue, and ganglion-like cysts, were compared between the two groups. The two groups exhibited significant differences regarding only three of these parameters, i.e., the presence of areas of chondroid metaplasia (Fig. 1), ganglion-like cysts (Fig. 2), and long septa (Fig. 3). The long septa, which were made up of bundles of collagen fiber with fibroblasts, traversed the elastic tissue in the LF. Cystic degeneration typically showed granulation tissue in the endocystic wall. The histopathological findings of both groups are summarized in Table 2.

Similarly, several parameters were assessed by transmission electron microscopy in the two groups. The

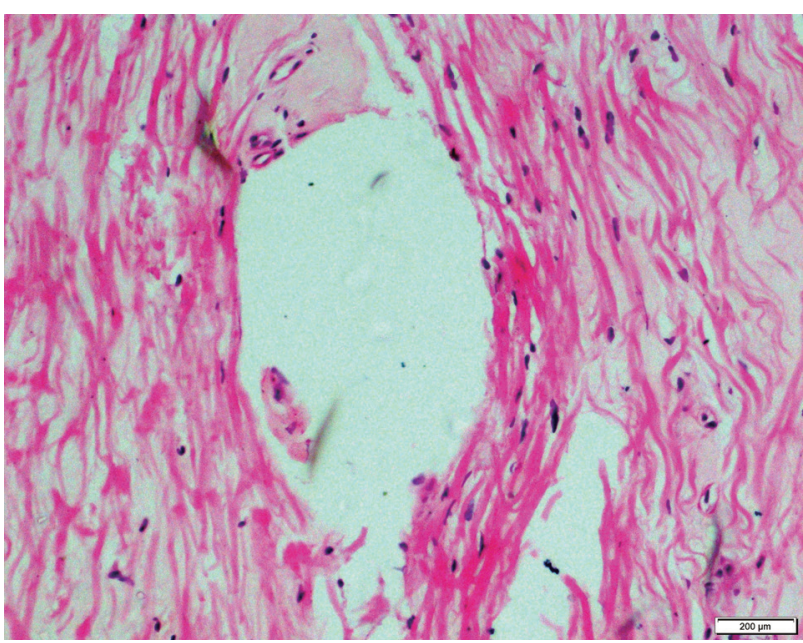

Fig. 2. HE stained section of ligamentum flavum of a patient with degenerative lumbar canal stenosis showing ganglion like cystic lesion $(H \& E, \times 100)$. $H E$, hematoxylin and eosin.

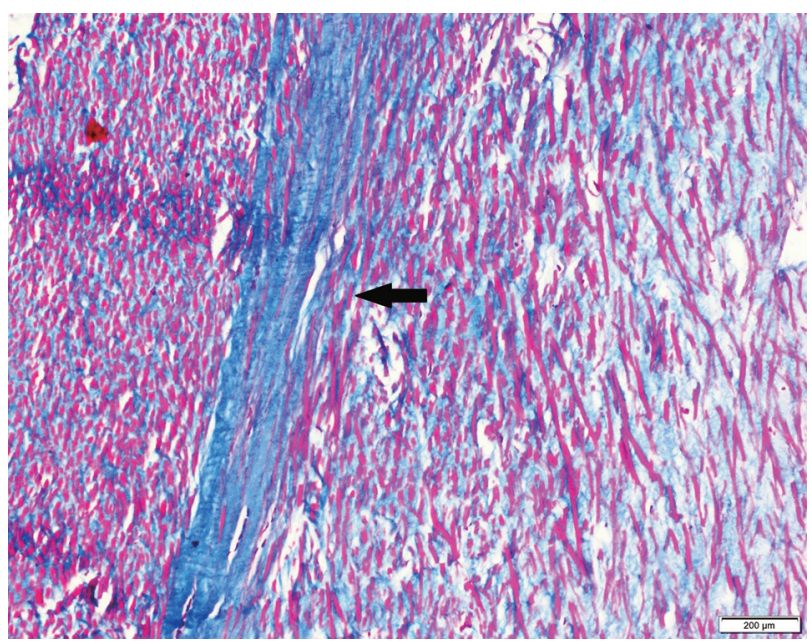

Fig. 3. Masson's trichrome-stained section of the ligamentum flavum in a patient with degenerative lumbar canal stenosis showing long septa of collagenous tissue traversing the elastic tissue (black arrow).

elastic fiber thickness, the quality of elastic fibers, the elastic:collagen ratio, presence of calcification, elaunin fibers, remnants of necrotic cells, and electron-dense material in the LF were evaluated. Significant differences between the two groups were observed for two of the parameters: the quality of elastic fibers and the elastic:collagen ratio. Patients with degenerative LCS exhibited significant thinning (Fig. 4), fragmentation, and degeneration of elastic fibers compared with patients with disk herniation. A summary of the findings obtained for both groups regarding each of these electron microscopic parameters is presented in Table 3. 

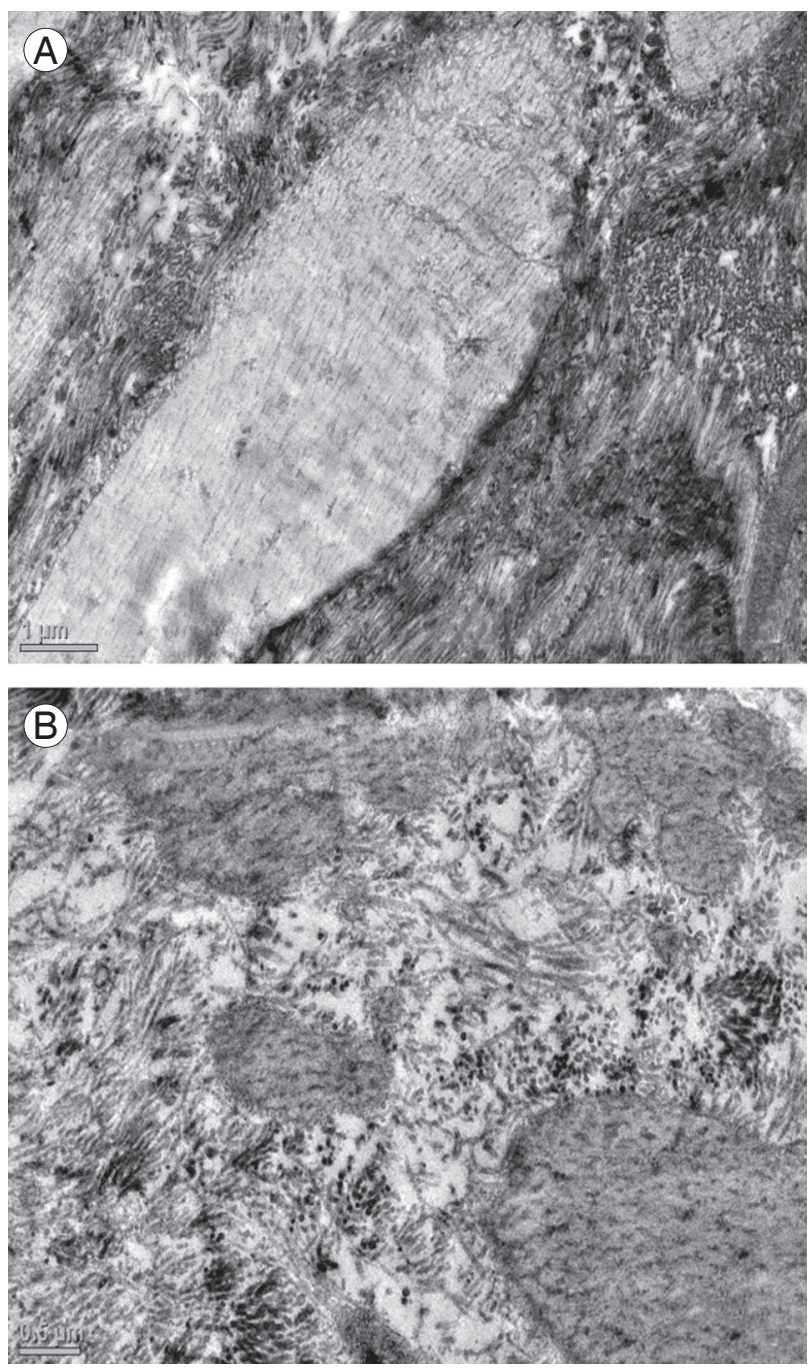

Fig. 4. (A) Electron micrograph of the ligamentum flavum in a patient of the control group showing normal elastic fibers $(\times 2,550)$ and $(\mathbf{B})$ in a patient with degenerative lumbar canal stenosis showing marked thinning of elastic fibers $(\times 4,000)$.

\section{Discussion}

Based on myelograms, computed tomography scans, magnetic resonance imaging, and intraoperative findings, many authors have thought that the alteration of the microenvironment in the LF is of the most important factors in the development of degenerative LCS $[5,6,12]$. The morphological changes in the LF that occur with aging have been described previously [11]. However, most of those studies date back to over a decade, a time at which the histological and electron microscopic techniques were not as refined as they are now. Many of these studies also have methodological pitfalls; therefore, we undertook the present study in an attempt to fill the void in the medical literature regarding the precise morphological changes in the LF in degenerative LCS.

The pioneering reports of Elsberg [3] and Park et al. [13] described the potential role of the LF in causing stenosis of the spinal canal, which is responsible for the classical symptom of neurological claudication observed in patients with degenerative LCS. There is ample histological evidence to prove that the LF is thicker $[13,14]$ and less elastic [11] and undergoes several characteristic global and focal changes that mark the underlying degenerative process $[6,15]$. However, considering the well-established role of the LF in the disease process, very few studies have explored the morphological alterations of this tissue in degenerative LCS.

In 1994, Postacchini et al. [11] studied the histological and ultrastructural characteristics of ligamentum flava obtained from three groups of patients: a disk herniation group, a LCS group, and a control group comprising patients who were operated for vertebral fractures. The authors found that, in the disk herniation group, the morphological changes mirrored those observed in patients of similar age compared with the control group. Areas of fibrosis, degenerating elastic fibers, and areas of calcification were detected more often in the lumbar stenosis group. However, the authors adopted a descriptive format to report their results and made no comment on whether any of these changes were statistically significant. Moreover, no objective quantification of the morphological changes was performed; hence, it is difficult to surmise the relevance of the changes described by those authors. A study from Iraq had a remarkably similar methodology and an analogous pattern of documentation of the results of the study, albeit with a much larger sample size [16]. In both of these studies, there was a great deal of overlap between the pathological conditions-patients with LDH in their fifth or sixth decade would naturally have degenerative changes and an added component of degenerative LCS.

Schräder et al. [5] compared the histological findings in ligamentum flava harvested from patients with lumbar stenosis with those harvested from fresh cadavers who did not have lumbar stenosis (as the control group). A limited number of characteristics were studied, i.e., the percentage of calcification and the number of elastic fibers, which were both significantly different between the two groups [5]. An attempt has also been made to correlate the histological findings of lumbar stenosis with clinical and 
Table 2. Summary of differences in various histopathological parameters in the two study groups

\begin{tabular}{|c|c|c|c|}
\hline Histopathological parameter & Degenerative lumbar canal stenosis (group 1) & Disc herniation (group 2) & $p$-value ${ }^{\text {a }}$ \\
\hline Fibrosis score & & & 0.27 \\
\hline Grade 1 fibrosis & $12 / 20$ & $12 / 20$ & \\
\hline Grade 2 fibrosis & $3 / 20$ & $6 / 20$ & \\
\hline Grade 3 fibrosis & $3 / 20$ & - & \\
\hline Grade 4 fibrosis & $1 / 20$ & - & \\
\hline No fibrosis & $1 / 20$ & $2 / 20$ & \\
\hline Percentage of median loss of elastic fibers ${ }^{b /}$ & $20 \%$ & $15 \%$ & 0.22 \\
\hline Percentage of calcification & & & 0.06 \\
\hline$<5 \%$ & $12 / 20$ & $5 / 20$ & \\
\hline$\geq 5 \%$ to $<10 \%$ & $2 / 20$ & $2 / 20$ & \\
\hline Absent & $6 / 20$ & $13 / 20$ & \\
\hline Areas of chondroid metaplasia & & & $0.03^{c \mid}$ \\
\hline Present at bony attachment & $15 / 20$ & $6 / 20$ & \\
\hline Present in central area & - & $1 / 20$ & \\
\hline Absent & $5 / 20$ & $13 / 20$ & \\
\hline Mucinous degeneration & & & 0.065 \\
\hline Present & $10 / 20$ & $7 / 20$ & \\
\hline Absent & $10 / 20$ & $13 / 20$ & \\
\hline Vascularization & & & 0.053 \\
\hline Present at bony attachment & $3 / 20$ & $10 / 20$ & \\
\hline Present at central area & $7 / 20$ & $6 / 20$ & \\
\hline Present in both central and at bony attachment & $3 / 20$ & - & \\
\hline Absent & $7 / 20$ & $4 / 20$ & \\
\hline Long septa & & & $0.004^{c)}$ \\
\hline Present & $15 / 20$ & $6 / 20$ & \\
\hline Absent & $5 / 20$ & $14 / 20$ & \\
\hline Clefts & & & 0.29 \\
\hline Present & $19 / 20$ & $17 / 20$ & \\
\hline Absent & $1 / 20$ & $3 / 20$ & \\
\hline Granulation tissue & & & 0.55 \\
\hline Present & $1 / 20$ & $2 / 20$ & \\
\hline Absent & $19 / 20$ & $18 / 20$ & \\
\hline Ganglion like cystic lesions & & & $0.004^{c)}$ \\
\hline Present & $4 / 20$ & - & \\
\hline Absent & $16 / 20$ & $20 / 20$ & \\
\hline
\end{tabular}

Values are presented as number/total number or \%, unless otherwise stated.

${ }^{a}$ Mann-Whitney test was used for percentage loss of fibers. For all other parameters, the chi-square test was used. ${ }^{b}$ The percentage loss of fibers was not in a normal distribution; hence, the medians were compared. ${ }^{\mathrm{c}}$ Statistically significant.

radiological findings, as reported by Okuda et al. [15]. The only significant correlation detected by these authors was that observed between the percentage of slippage of the vertebra, as assessed radiologically, and the ossification of the LF, as assessed histopathologically. In addition to the number of elastic fibers and their degeneration noted in earlier studies, the authors have recorded a host of other focal findings, including the presence of ganglion- 
Table 3. Summary of differences in various electron microscopic parameters in the two study groups

\begin{tabular}{|c|c|c|c|}
\hline Electron microscopic parameter & Degenerative lumbar canal stenosis (group 1) & Disc herniation (group 2) & $p$-value ${ }^{a}$ \\
\hline Thickness of elastic fibers $(\mu \mathrm{m})$ & $3.196 \pm 0.8216$ & $3.518 \pm 0.8384$ & 0.322 \\
\hline Degeneration of elastic fibers & & & $0.000^{\mathrm{bl}}$ \\
\hline Present & $16 / 20$ & $3 / 20$ & \\
\hline Absent & $4 / 20$ & $17 / 20$ & \\
\hline Median elastic: collagen ratioc) & 0.07 & 1.20 & $0.023^{b /}$ \\
\hline Elaunin fibers & & & 1.000 \\
\hline Present & $2 / 20$ & $2 / 20$ & \\
\hline Absent & $18 / 20$ & $18 / 20$ & \\
\hline Remnants of necrotic cells & & & 0.091 \\
\hline Present & $9 / 20$ & $4 / 20$ & \\
\hline Absent & $11 / 20$ & $16 / 20$ & \\
\hline Electron dense material & & & 0.548 \\
\hline Present & $2 / 20$ & $1 / 20$ & \\
\hline Absent & $18 / 20$ & $19 / 20$ & \\
\hline Calcification & & & 0.376 \\
\hline Present & $4 / 20$ & $2 / 20$ & \\
\hline Absent & $16 / 20$ & $18 / 20$ & \\
\hline
\end{tabular}

Values are presented as mean \pm standard deviation, number/total number, or median, unless otherwise stated.

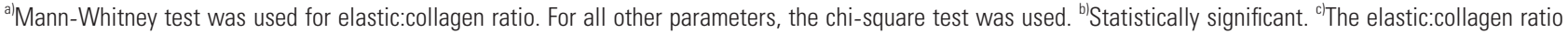
was not in a normal distribution; hence, the medians were compared.

like cysts, vascularization, mucinous degeneration, ossification, calcification, and chondrogenesis. Differences in some of these morphological changes have also been noted between the different layers of the LF-the dorsal layers exhibited more fibrosis and a more significant decrease in the number of elastic fibers compared with the dural layer [17]. Kosaka et al. [6] performed a histological, biological, and immunohistochemical assessment of human samples of ligamentum flava in which they noted a similar tendency toward the occurrence of the morphological changes that are representative of degeneration more frequently on the dorsal aspect of the LF compared with the dural aspect. The samples were divided into three age groups: those acquired from fetuses (single cadaveric sample), young patients, and elderly patients. An age-related decrease in elastic fibers with a concomitant increase in collagen fibers was noted. The only recent study on this topic was performed in Turkey and involved the histopathological examination of the LF in patients with LDH and lumbar spinal stenosis [18]. Significant differences were noted with reference to two characteristics, i.e., the mean elastic fiber alignment and the mean calcification.

Our study had several unique aspects in its methodol- ogy that we believe are an improvement over previous studies. We performed detailed electron microscopic and histopathological examinations and analyzed several characteristics that had not been noted previously. All patients were prospectively recruited and, thus, the standardized techniques outlined in the protocol were used for sample preparation. Our study had a control group that comprised young patients (age $<35$ years) with $\mathrm{LDH}$, whereas the study group included older patients (age $>55$ years) with degenerative LCS. There was no overlap between the two groups. This was unlike that described in previous studies, in which the elderly patients in the disk herniation group may have had a component of degenerative LCS, thus hampering the interpretation of the results. The histopathological examination revealed that a significantly greater number of patients with degenerative LCS exhibited areas of chondroid metaplasia, ganglion-like cystic lesions, and long septa compared with the LDH group. However, there were no significant differences between the two groups in terms of fibrosis score, loss of elastic fibers, degeneration of elastic fibers, percentage of calcification, presence of vascularization, mucinous degeneration, granulation tissue, and clefts. In turn, the electron 
microscopic examination showed that the degenerative LCS group had a significantly lower elastic:collagen ratio and that a significantly greater number of patients in this group had degeneration of elastic fibers compared with the control group. However, there were no significant differences between the two groups in terms of thickness of elastic fibers, presence of calcification, remnants of necrotic cells, elaunin fibers, and electron-dense material. We did not perform a layer-by-layer examination of the LFWhile this study is essentially a pilot study in this part of the world, where no such study had been performed previously, we recognize certain limitations. The sample size was small considering the prevalence of degenerative LCS. However, the time-consuming and resourceintensive nature of the study only allowed the recruitment of 20 patients into each group. Unlike the study reported by Okuda et al. [15], we did not perform any clinical and radiological correlations with the histological findings. It is common knowledge that the symptoms of degenerative spine pathology are often disproportionate to the underlying extent of the disease; thus, investigating for the presence of such a correlation would be of great benefit. There were also some technical shortcomings in the way we procured the specimens of the LF and performed the sectioning for histopathological and electron microscopy examination. The LF could not be removed en-bloc in most cases; therefore, differences in the characteristics examined among the different parts of the LF (dorsal/central/central/bony attachment) could not be assessed.

\section{Conclusions}

We conclude that the presence of areas of chondroid metaplasia, ganglion-like cystic lesions, and long septa according to histopathological examination and a lower elastic:collagen ratio and the presence of degeneration of elastic fibers according to electron microscopic examination are indicative degenerative changes in the LF. If validated by multi-centric and multi-ethnic studies with a larger sample size, these parameters can be the focus of novel diagnostic and therapeutic strategies to treat degenerative LCS.

\section{Conflict of Interest}

No potential conflict of interest relevant to this article was reported.

\section{Acknowledgments}

This study has been partially funded by a grant received from the Indian Council of Medical Research. We would like to thank Dr. Rimlee Dutt, Senior Resident (Pathology) for her help in histopathological examination of samples as a part of the study.

\section{Author Contributions}

Study design and concept: DH, BG, ARM, TCN, KF; data collection and data analysis: DH, NM, BG; review of literature: $\mathrm{DH}, \mathrm{NM}, \mathrm{BG}, \mathrm{KF}$; manuscript preparation: $\mathrm{DH}$, $\mathrm{NM}, \mathrm{BG}, \mathrm{KF}$; and editing and proof reading: $\mathrm{DH}, \mathrm{NM}$, BG, KF, TCN, ARM

\section{References}

1. Deyo RA, Gray DT, Kreuter W, Mirza S, Martin BI. United States trends in lumbar fusion surgery for degenerative conditions. Spine (Phila Pa 1976) 2005;30:1441-7.

2. Yabuki S, Fukumori N, Takegami M, et al. Prevalence of lumbar spinal stenosis, using the diagnostic support tool, and correlated factors in Japan: a population-based study. J Orthop Sci 2013;18:893-900.

3. Elsberg CA. Experiences in spinal surgery: observations upon 60 laminectomies for spinal disease. Surg Gynecol Obstet 1913;16:117-32.

4. Fukuyama S, Nakamura T, Ikeda T, Takagi K. The effect of mechanical stress on hypertrophy of the lumbar ligamentum flavum. J Spinal Disord 1995;8:12630.

5. Schrader PK, Grob D, Rahn BA, Cordey J, Dvorak J. Histology of the ligamentum flavum in patients with degenerative lumbar spinal stenosis. Eur Spine J 1999;8:323-8.

6. Kosaka H, Sairyo K, Biyani A, et al. Pathomechanism of loss of elasticity and hypertrophy of lumbar ligamentum flavum in elderly patients with lumbar spinal canal stenosis. Spine (Phila Pa 1976) 2007;32:2805-11.

7. Viejo-Fuertes D, Liguoro D, Rivel J, Midy D, Guerin J. Morphologic and histologic study of the ligamentum flavum in the thoraco-lumbar region. Surg Radiol Anat 1998;20:171-6.

8. Ramsey RH. The anatomy of the ligamenta flava. Clin 
Orthop Relat Res 1966;44:129-40.

9. Baba H, Maezawa Y, Furusawa N, Imura S, Tomita $\mathrm{K}$. The role of calcium deposition in the ligamentum flavum causing a cauda equina syndrome and lumbar radiculopathy. Paraplegia 1995;33:219-23.

10. Vernet O, Fankhauser H, Schnyder P, Deruaz JP. Cyst of the ligamentum flavum: report of six cases. Neurosurgery 1991;29:277-83.

11. Postacchini F, Gumina S, Cinotti G, Perugia D, DeMartino C. Ligamenta flava in lumbar disc herniation and spinal stenosis: light and electron microscopic morphology. Spine (Phila Pa 1976) 1994;19:917-22.

12. Grenier N, Kressel HY, Schiebler ML, Grossman RI, Dalinka MK. Normal and degenerative posterior spinal structures: MR imaging. Radiology 1987;165:51725.

13. Park JB, Chang H, Lee JK. Quantitative analysis of transforming growth factor-beta 1 in ligamentum flavum of lumbar spinal stenosis and disc herniation.
Spine (Phila Pa 1976) 2001;26:E492-5.

14. Abbas J, Hamoud K, Masharawi YM, et al. Ligamentum flavum thickness in normal and stenotic lumbar spines. Spine (Phila Pa 1976) 2010;35:1225-30.

15. Okuda T, Baba I, Fujimoto Y, et al. The pathology of ligamentum flavum in degenerative lumbar disease. Spine (Phila Pa 1976) 2004;29:1689-97.

16. Hatem HA, Jbara KK, Hamdan TA. Histological changes of ligamenta flava in lumbar disc herniation and spinal canal stenosis. Basrah J Surg 2005;11:2437.

17. Sairyo K, Biyani A, Goel V, et al. Pathomechanism of ligamentum flavum hypertrophy: a multidisciplinary investigation based on clinical, biomechanical, histologic, and biologic assessments. Spine (Phila Pa 1976) 2005;30:2649-56.

18. Altun I, Yuksel KZ. Histopathological analysis of ligamentum flavum in lumbar spinal stenosis and disc herniation. Asian Spine J 2017;11:71-4. 